\title{
IncRNA DGCR5 acts as a tumor suppressor in papillary thyroid carcinoma via sequestering miR-2861
}

\author{
FUKUN CHEN $^{1,2}$, SHUTING YIN $^{3}$, JIALUN ZHU ${ }^{1,2}$, PENGJIE LIU $^{1,2}$, \\ CHUANZHOU YANG ${ }^{1,2}$, ZHIPING FENG ${ }^{1,2}$ and ZHIYONG DENG ${ }^{1,2}$
}

\begin{abstract}
${ }^{1}$ Department of Nuclear Medicine, Yunnan Tumor Hospital; ${ }^{2}$ Department of Nuclear Medicine, The Third Affiliated Hospital of Kunming Medical University, Kunming, Yunnan 650118; ${ }^{3}$ Third Ward of The Department of Urology, The Second Affiliated Hospital of Kunming Medical University, Kunming, Yunnan 650000, P.R. China
\end{abstract}

Received June 8, 2018; Accepted September 13, 2018

DOI: $10.3892 / \mathrm{etm} .2018 .7012$

\begin{abstract}
A vast amount of evidence indicates that long non-coding RNAs (lncRNAs) are involved in cancer. Previous studies have indicated that lncRNA DiGeorge syndrome critical region gene 5 (DGCR5) is aberrantly expressed in lung cancer, pancreatic ductal adenocarcinoma and hepatocellular carcinoma. However, the role of DGCR5 in papillary thyroid carcinoma (PTC) has remained elusive. In the present study, it was revealed that DGCR5 was significantly downregulated in PTC tissues compared with that in adjacent normal tissues. Through functional experiments, it was demonstrated that ectopic overexpression of DGCR5 markedly suppressed PTC cell growth and invasion. A bioinformatics analysis suggested that DGCR5 binds to microRNA (miR)-2861. A total of 5 putative binding sites for miR-2861 were identified in DGCR5, and a luciferase reporter assay confirmed the direct interaction between DGCR5 and miR-2861. Furthermore, reverse transcription-quantitative polymerase chain reaction analysis indicated that ectopic overexpression of DGCR5 led to a decreased expression of miR-2861 in PTC cells and miR-2861 mimic transfection caused a downregulation of DGCR5. miR-2861 level was upregulated in PTC tissues compared with adjacent tissues and negatively correlated with DGCR5 level. In addition, rescue experiments indicated that ectopic expression of miR-2861 reversed the effects of DGCR5 overexpression on PTC cell proliferation and invasion. Taken together, the present results demonstrated that DGCR5 inhibits PTC progression via sponging miR-2861, indicating DGCR5 may serve as a therapeutic target.
\end{abstract}

Correspondence to: Dr Zhiyong Deng or Dr Zhiping Feng, Department of Nuclear Medicine, Yunnan Tumor Hospital, 519 Kunzhou Road, Kunming, Yunnan 650118, P.R. China

E-mail: dengzhiyongjz@163.com

E-mail: fengzhipingqq@163.com

Key words: papillary thyroid carcinoma, long noncoding RNA, DiGeorge syndrome critical region gene 5, proliferation, invasion

\section{Introduction}

Thyroid carcinoma (TC) is one of the most prevalent cancer types of the endocrine system and its incidence is gradually increasing (1). According to histologic features, TC may be classified into four types, namely papillary, medullary, follicular and anaplastic TC (2). Among all cases, papillary TC (PTC) accounts for $80 \%$ of malignancies (3). Although the outcomes for patients with early-stage PTC are favorable, the five-year survival rate for patients with PTC in the advanced stage remains low (4). Thus, it is crucial to understand the molecular mechanisms of PTC progression and identify more effective therapeutic strategies.

Long non-coding RNAs (lncRNAs) are a group of non-coding RNAs of $>200$ nucleotides in length (5). IncRNAs have no protein-coding ability and account for a large proportion of genomic transcripts. A vast amount of evidence has indicated that lncRNAs act as modulators in tumor progression (6). IncRNAs have been reported to participate in multiple pathological processes in cancer, including cell survival, proliferation and epithelial-mesenchymal transition (7). Numerous lncRNAs have been observed to be aberrantly expressed in the tissues and to be implicated in the progression of various types of tumor. For instance, lncRNA mucin (MUC)5B-antisense non-coding RNA 1 enhances lung adenocarcinoma metastasis by regulating MUC5B expression (8). Overexpression of lncRNA DQ786243 predicts poor prognosis and contributes to the progression of hepatocellular carcinoma (HCC) (9). Cytoskeleton regulator RNA enhances glioma development via sponging microRNA (miR)-16 (10). These results support the important roles of lncRNAs in cancer.

A previous study revealed that lncRNA DiGeorge syndrome critical region gene 5 (DGCR5) is a suppressor of lung cancer, HCC and pancreatic ductal adenocarcinoma progression (11-13). However, the role of DGCR5 in PTC has remained elusive. The present study therefore aimed to investigate the role of DGCR5 in PTC progression and to determine the underlying mechanisms. It was demonstrated that DGCR5 suppressed PTC cell proliferation and invasion through acting as a sponge for miR-2861. The present study suggested that DGCR5 may be a promising therapeutic target for PTC. 


\section{Materials and methods}

Patients and samples. A total of 32 PTC samples (14 males and 18 females; age range, 37-61 years old; median age, 52 years old) and adjacent non-cancerous tissues ( $2 \mathrm{~cm}$ away from the lesion) were obtained from Yunnan Tumor Hospital (Kunming, China) between September 2014 and October 2016. All tissues were frozen in liquid nitrogen and stored at $-80^{\circ} \mathrm{C}$ after the surgery. The current study was approved by the Ethics Committee on Human Research of Yunnan Tumor Hospital (Kunming, China). Written informed consent was provided by all patients.

Cell culture. The TPC1 PTC cell line and the Nthyori3-1 human thyroid epithelial cell line were purchased from the Cell Bank of The Chinese Academy of Sciences (Beijing, China) and cultured in RPMI-1640 medium supplemented with 10\% fetal bovine serum (FBS; Gibco; Thermo Fisher Scientific, Inc., Waltham, MA, USA), $100 \mathrm{U} / \mathrm{ml}$ penicillin and $100 \mathrm{mg} / \mathrm{ml}$ streptomycin (Gibco; Thermo Fisher Scientific, Inc.) at $37^{\circ} \mathrm{C}$ in a humidified atmosphere containing $5 \% \mathrm{CO}_{2}$.

Transient transfection. Cells in 6-well dishes were transfected with pcDNA3.1-DGCR5 $(2 \mu \mathrm{g})$, pcDNA3.1-vector (Addgene, Inc., Cambridge, MA, USA), miR-2861 mimics (50 nM, 5'-GGGGCCUGGCGGUGGGCGG-3') or negative control mimics (50 nM, 5'-UCACAACCUCCUAGAAAG AGUAGA-3'; both Shanghai GenePharma Co., Ltd. Shanghai, China) using Lipofectamine 2000 (Invitrogen; Thermo Fisher Scientific, Inc.) according to the manufacturer's protocols.

Reverse transcription quantitative polymerase chain reaction $(R T-q P C R)$. RNA isolation from homogenized tissues or cells was performed using TRIzol reagent (Invitrogen; Thermo Fisher Scientific, Inc.). Extracted RNA $(1 \mu \mathrm{g})$ was reverse-transcribed into complementary DNA using the QuantiTect Reverse Transcription Kit (cat. no. 205313; Qiagen, Valencia, CA, USA). PCR was performed using SYBR-Green Premix Ex Taq II (cat. no. RR820A; Takara, Otsu, Japan) on an Applied Biosystems Step One Plus Real-Time PCR System (Applied Biosystems, Thermo Fisher Scientific, Inc.). The thermocycling conditions were as follows: Initial denaturation at $95^{\circ} \mathrm{C}$ for $10 \mathrm{~min}$; followed by 40 cycles of denaturation at $95^{\circ} \mathrm{C}$ for $15 \mathrm{sec}$ and elongation at $60^{\circ} \mathrm{C}$ for $1 \mathrm{~min}$. Gene expression levels were normalized to the levels of U6 and quantified according to the $2^{-\Delta \Delta \mathrm{Cq}}$ method (14). Primer sequences are listed in Table I. Melting curve analysis was conducted at $95^{\circ} \mathrm{C}$ for $15 \mathrm{sec}, 60^{\circ} \mathrm{C}$ for $1 \mathrm{~min}$ and $95^{\circ} \mathrm{C}$ for $15 \mathrm{sec}$ for quantification of the results.

Cell Counting Kit (CCK)8 proliferation assay. Cells were seeded into a 96 -well plate at $2 \times 10^{3}$ cells/well and cultured for 24, 48 or $72 \mathrm{~h}$. Subsequently, $10 \mu \mathrm{l} \mathrm{CCK}-8$ stain (Beyotime, Shanghai, China) was added to each well, followed by incubation for $2 \mathrm{~h}$ at $37^{\circ} \mathrm{C}$ and determination of the absorbance at $450 \mathrm{~nm}$ using an ELx808 microplate reader (BioTek Instruments, Inc., Winooski, VT, USA).

Transwell assay. For the invasion assay, $2 \times 10^{4}$ cells in $200 \mu \mathrm{l}$ serum-free medium were placed in each of the upper chambers of a Transwell plate ( $8 \mu \mathrm{m}$ pore; Corning, Inc., Corning, NY, USA) whose membrane filters had been pre-coated with Matrige ${ }^{\circledR}$ (Corning, Inc.). The lower chambers were filled with $600 \mu \mathrm{l}$ medium containing 10\% FBS. After incubation for $24 \mathrm{~h}$, cells on the upper side were wiped away and the cells that had moved into the lower side of the membrane were fixed with methanol for $30 \mathrm{~min}$ at $25^{\circ} \mathrm{C}$ and stained with $0.1 \%$ crystal violet for $30 \mathrm{~min}$ at $25^{\circ} \mathrm{C}$. The cells were observed through an Olympus microscope (Olympus Corp., Tokyo, Japan) and the number of cells was counted in five randomly selected fields. The results were expressed as the average number of invaded cells in each field.

Luciferase assay. The potential targets of DGCR5 were predicted using the miRDB tool (http://mirdb. org/miRDB/index.html). Then DGCR5 sequences containing the wild-type (WT) binding site for miR-2861, the cDNA of which was obtained from the aforementioned RT-qPCR analysis, was amplified by PCR. The mutant (Mut) binding site was synthesized by Sangon Biotech Co., Ltd. (Shanghai, China). These sequences were cloned into pmiR-GLO vector (Promega Corporation, Madison, WI, USA) to obtain reporter vector. For luciferase reporter assay, miR-2861 or scramble mimics and WT or Mut of DGCR5 fragment were co-transfected into $1 \times 10^{5}$ TPC1 cells along with 2 ng Renilla luciferase herpes simplex virus tyrosine kinase plasmid (Promega Corp., Madison, WI, USA). Luciferase activity was detected using the Dual-Luciferase Reporter Assay System (Promega Corp.) according to the manufacturer's protocols, and the ratio of Firefly to Renilla luciferase activity was determined.

Statistical analysis. SPSS version 19 (IBM Corp., Armonk, NY, USA) or GraphPad Prism 5.0 (GraphPad Software, Inc, La Jolla, CA, USA) was used for data analysis. All results were expressed as the mean \pm standard deviation. Student's t-test or one-way analysis of variance followed by Tukey's post-hoc test were utilized to determine the statistical significance of differences between groups. Spearman's rank correlation analysis was used to determine the correlation between miR-2861 and DGCR5 expression. $\mathrm{P}<0.05$ was considered to indicate a statistically significant difference.

\section{Results}

DGCR5 is downregulated in PTC tissues. To identify the potential function of DGCR5 in PTC progression, the levels of DGCR5 were measured in 32 pairs of PTC tissues and adjacent normal tissues by RT-qPCR. The results indicated that DGCR5 was significantly downregulated in PTC tissues compared with that in adjacent normal tissues (Fig. 1A). Similarly, RT-qPCR analysis was used to assess the levels of DGCR5 in the TPC1 PTC cell line and in the Nthyori 3-1 normal thyroid cell line. The results indicated that DGCR5 was also downregulated in the PTC cell line compared with that in the normal cell line (Fig. 1B). These results suggested that DGCR5 may be a tumor suppressor in PTC.

DGCR5 overexpression inhibits PTC cell proliferation and invasion. The present study further sought to explore the roles of DGCR5 in PTC. Thus, DGCR5 was overexpressed in TPC1 cells 
Table I. Primer sequences for polymerase chain reaction.

Primer (5'-3')

$\begin{array}{llr}\text { Gene } & \text { Forward } & \text { Reverse }\end{array}$

DGCR5

miR-2861

U6

\section{CCAAGCCTGTCTGTGTGTTC \\ AACGAGACGACGACAGAC \\ CTCGCTTCGGCAGCACA}

GGGAGACACAGACCACAAGA

GGGGCCUGGCGGUGGGCGG

AACGCTTCACGAATTTGCGT

DGCR5, DiGeorge syndrome critical region gene 5; miR, microRNA.
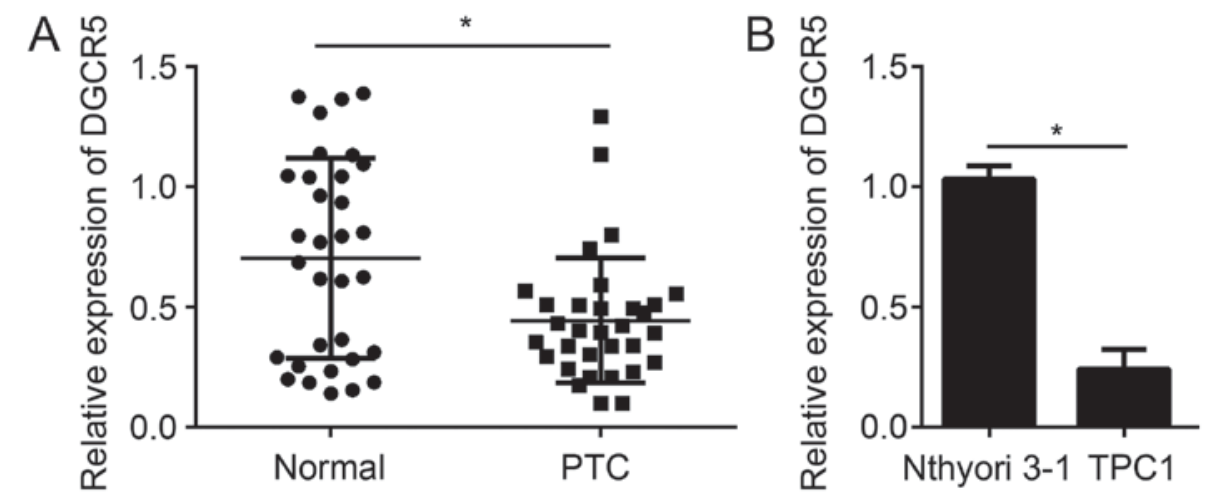

Figure 1. DGCR5 is downregulated in PTC tissues. (A) Relative expression levels of DGCR5 were determined by RT-qPCR in 32 pairs of PTC tissues and normal tissues. (B) The expression of DGCR5 in PTC cell lines was examined by RT-qPCR. "P $<0.05$ vs. control group. PTC, papillary thyroid carcinoma; DGCR5, DiGeorge syndrome critical region gene 5; RT-qPCR, reverse transcription-quantitative polymerase chain reaction.

via transfection with pcDNA3.1-DGCR5. RT-qPCR analysis revealed that overexpression of DGCR5 significantly promoted DGCR5 expression compared with that in the control-transfected cells (Fig. 2A). Subsequently, the cells were subjected to CCK8 and Transwell invasion assays to determine the effect of DGCR5 on PTC cell growth and invasion. The results indicated that ectopic expression of DGCR5 markedly inhibited the proliferation and invasion of TPC1 cells (Fig. 2B and C).

DGCR5 sequesters miR-2861. To determine the molecular mechanisms of the roles of DGCR5 in PTC, potential target miRs of DGCR5 were predicted by a bioinformatics analysis. Among the miRs identified, miR-2861 stood out, as it had five potential binding sites for DGCR5 (Fig. 3A). Of note, transfection of miR-2861 mimics significantly reduced the levels of DGCR5 in TPC1 cells compared with that in negative control-transfected cells (Fig. 3B). Consistently, overexpression of DGCR5 also led to decreased levels of miR-2861 in TPC1 cells (Fig. 3C), indicating a close association between DGCR5 and miR-2861. To further confirm the direct interaction of DGCR5 and miR-2861, a luciferase reporter assay was performed. The results revealed that ectopic expression of miR-2861 significantly repressed the luciferase activity of DGCR5-WT, but not that ofDGCR5-Mutin TPC1 cells(Fig.3D). These results suggested that DGCR5 sequesters miR-2861 in TPC1 cells.

Correlation of DGCR5 and miR-2861 levels in PTC. Next, the levels of miR-2861 in PTC tissues were measured by
RT-qPCR. The results illustrated that the miR-2861 levels in PTC tissues were higher than those in normal tissues (Fig. 4A). Furthermore, the levels of DGCR5 were inversely correlated with those of miR-2861 in PTC tissues (Fig. 4B), further supporting that DGCR5 serves as a miR sponge for miR-2861.

miR-2861 mimics abrogate the effects of DGCR5 overexpression. The above results demonstrated that DGCR5 regulates miR-2861 levels in PTC cells. Therefore, the present study then sought to determine whether miR-2861 is involved in DGCR5-mediated regulation of PTC cells. A CCK8 and a Transwell invasion assay were performed, and the results indicated that overexpression of DGCR5 inhibited TPC1 cell proliferation and invasion, whereas co-transfection with miR-2861 mimics significantly abrogated this effect (Fig. 5A and B). Taken together, it was demonstrated that DGCR5 acts as a tumor suppressor via sequestering miR-2861.

\section{Discussion}

Initially, lncRNAs were assumed to represent transcriptional noise with no cellular function. However, it was then demonstrated that lncRNAs possess essential functions in various biological processes, including the regulation of development, the immune response and tumorigenesis $(15,16)$. In cancer, lncRNAs have been proven to affect numerous important cellular processes, including proliferation, apoptosis, invasion and the cell cycle (17). 

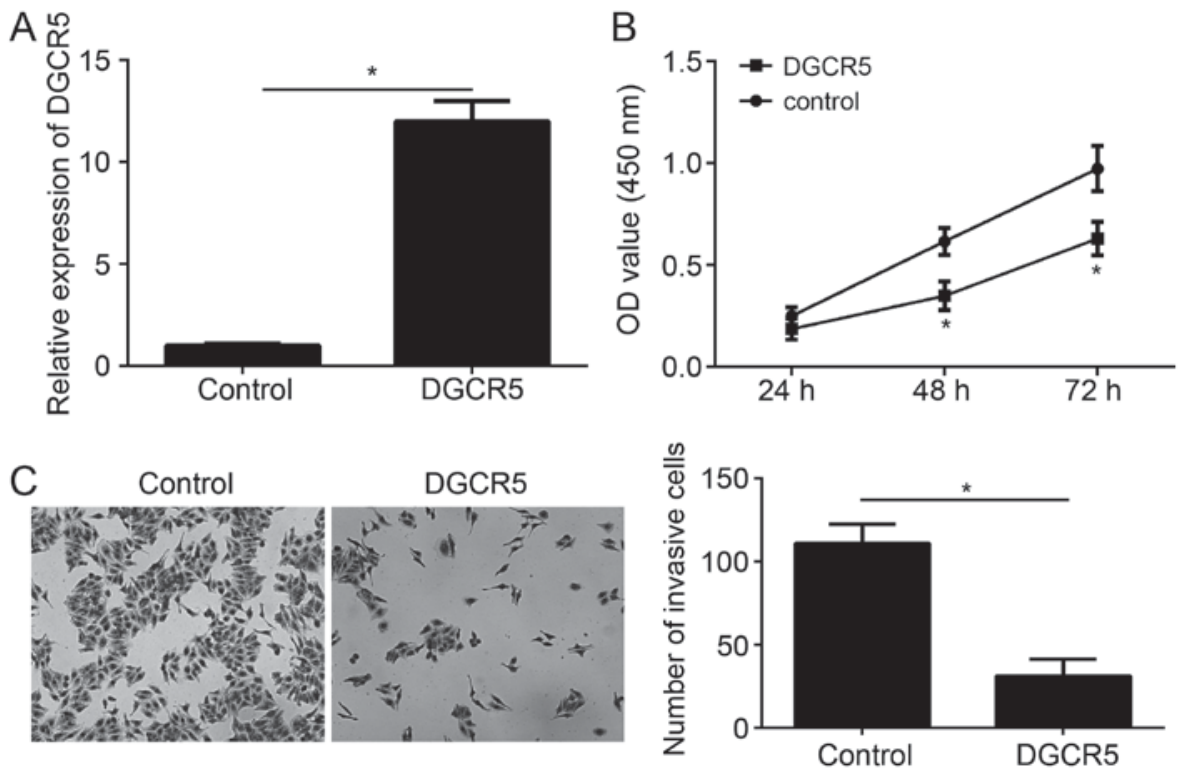

Figure 2. DGCR5 overexpression inhibits papillary thyroid carcinoma cell proliferation and invasion. (A) DGCR5 expression levels were significantly upregulated in TPC1 cells transfected with pcDNA1-DGCR5 plasmid. (B) A Cell Counting Kit-8 assay indicated that DGCR5 overexpression inhibited TPC1 cell proliferation. (C) A Transwell invasion assay indicated that DGCR5 overexpression inhibited TPC1 cell invasion (magnification, $\mathrm{x} 100)$. ${ }^{*} \mathrm{P}<0.05 \mathrm{vs.}$ control group. DGCR5, DiGeorge syndrome critical region gene 5; OD, optical density.

A

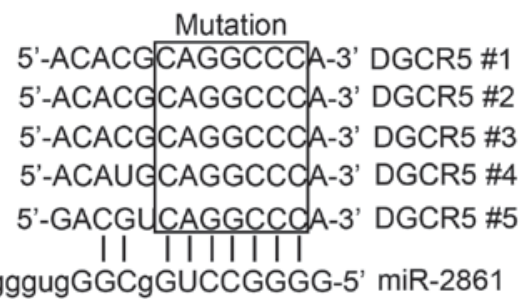

3'-gcgggugGGCgGUCCGGGG-5' miR-2861

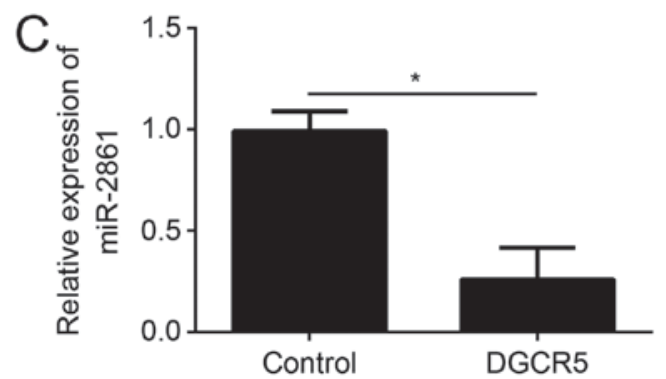

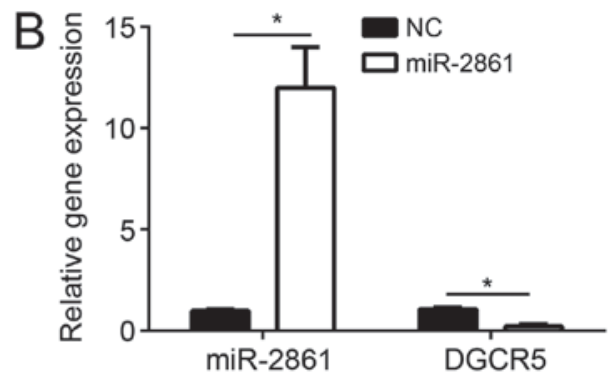

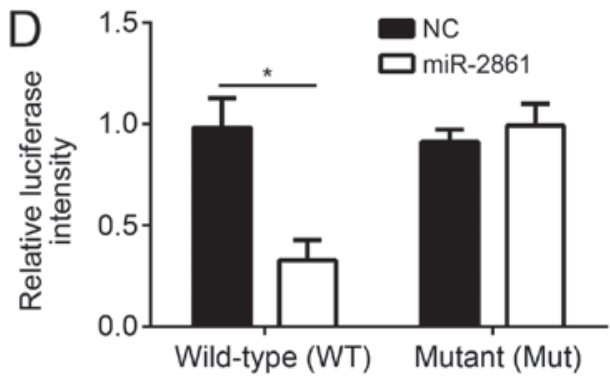

Figure 3. DGCR5 serves as a sponge for miR-2861. (A) Predicted miR-2861 binding sites in DGCR5. (B) Overexpression of miR-2861 suppressed DGCR5 expression in TPC1 cells. (C) Overexpression of DGCR5 inhibited the expression of miR-2861 in TPC1 cells. (D) A luciferase reporter assay indicated that overexpression of miR-2861 repressed the luciferase activity of DGCR5-WT, but not that of DGCR5-Mut, in TPC1 cells. "P<0.05 vs. control group. miR, microRNA; WT, wild-type; Mut, mutated; DGCR5, DiGeorge syndrome critical region gene 5; NC, mimic negative control.

lncRNA has been reported to be aberrantly expressed and involved in PTC progression (18). For instance, lncRNA maternally expressed 3 inhibits PTC cell invasion through suppressing Rac family small GTPase 1 (19). lncRNA AB074169 is downregulated and suppresses cell proliferation via modulation of KH-type splicing regulatory protein-mediated p21 expression in PTC (20). lncRNA small nucleolar RNA host gene 12 promotes PTC progression by activating the Wnt/ $\beta$-catenin axis (21). IncRNA PTC susceptibility candidate 3 contributes to PTC development and progression through inhibiting miR-574-5p (22). lncRNA HIT000218960 promotes PTC cell proliferation by increasing high mobility group AT-hook 2 expression (23). Overexpression of IncRNA NR_036575.1 was reported to promote PTC progression (24).

As for DGCR5, its function has remained to be fully elucidated. Wang et al (11) reported that DGCR5 promotes non-small cell lung cancer cell stemness by regulating miR-330-5p/CD44 signaling. A study by Chen et al (25) suggested that DGCR5 promotes lung cancer progression 

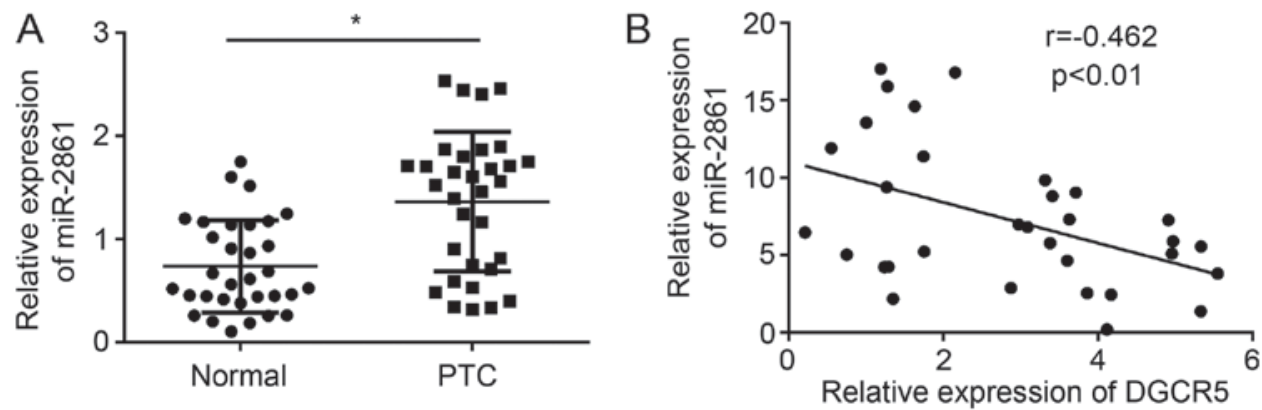

Figure 4. Association between DGCR5 and miR-2861 expression. (A) miR-2861 expression was significantly upregulated in PTC tissues compared with that in adjacent normal tissues. (B) The expression of DGCR5 was inversely correlated with that of miR-2861 in PTC tissues. "P $<0.05$ vs. control group. DGCR5, DiGeorge syndrome critical region gene 5; miR, microRNA; PTC, papillary thyroid carcinoma.

A

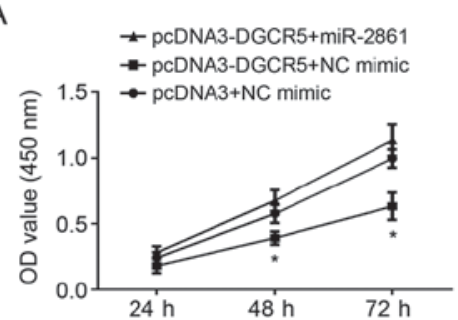

B

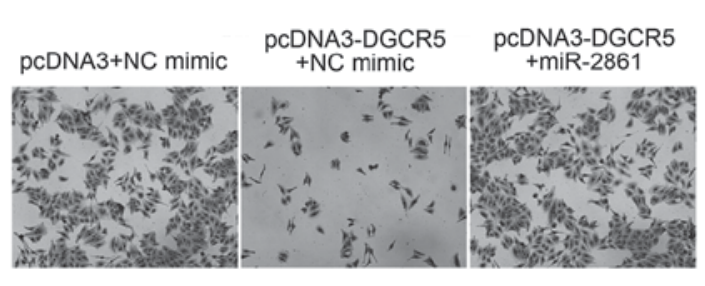

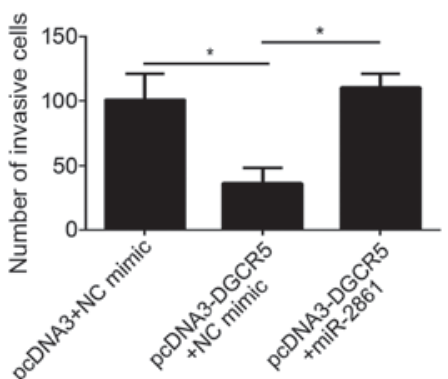

Figure 5. Ectopic expression of miR-2861 abrogates the effects of DGCR5 overexpression. (A) A Cell Counting Kit- 8 assay was used to determine the proliferation of TPC1 cells transfected with the indicated plasmids. (B) A Transwell invasion assay was utilized to determine the invasion of TPC1 cells transfected with the indicated plasmids (magnification, x100). "P<0.05 vs. pcDNA3+NC mimic. DGCR5, DiGeorge syndrome critical region gene 5; miR, microRNA; OD, optical density.

through sponging miR-1180. In HCC, downregulation of DGCR5 was associated with poor prognosis (13). In addition, Yong et al (12) indicated that DGCR5 is involved in regulating the malignant behavior of pancreatic ductal adenocarcinoma. However, the possible role of DGCR5 and PTC and its progression has remained elusive. To the best of our knowledge, the present study was the first to investigate this issue.

The present study demonstrated that DGCR5 expression was significantly downregulated in 32 PTC tissues compared with that in adjacent normal tissues. A CCK8 and a Transwell invasion assay revealed that ectopic overexpression of DGCR5 significantly suppressed the proliferation and invasion of TPC1 cells. lncRNAs may serve as competing endogenous (ce)RNAs for miRs (26) and previously, DGCR5 was also reported to be a ceRNA in lung cancer (25). Thus, it was further explored whether DGCR5 has a similar mechanism of action in PTC. Through a bioinformatics analysis, miR-2861 was identified as a target of DGCR5. The direct interaction of DGCR5 and miR-2861 was demonstrated by a luciferase reporter assay. miR-2861 overexpression has previously reported to be associated with PTC metastasis (27). Other studies have indicated that miR-2861 is involved in the development of lung and cervical cancer $(28,29)$. The present results indicate that miR-2861 is upregulated in PTC tissues compared with that in adjacent normal tissues, also suggesting that miR-2861 may serve as an oncogene in PTC. Furthermore, rescue experiments demonstrated that miR-2861 overexpression significantly promoted the proliferation and invasion of TPC1 cells transfected with pcDNA3.1-DGCR5.
However, the present study has certain limitations. The mechanism via which DGCR5 expression is regulated in PTC remains elusive. Furthermore, the downstream targets of the DGCR 5/miR-2861 axis still require to be elucidated. In addition, it remains to be determined whether DGCR5 regulates any further miRs in PTC. The authors of the current study only used a small number of samples. In the future, more samples were required to determine the association between the expression level of DGCR5 or miR-2861 with tumor clinical features, including tumor stage and metastasis.

\section{Acknowledgements}

Not applicable.

\section{Funding}

The present study was supported by Yunnan Science and Technology Project [grant no. 2017FE467(-080)].

\section{Availability of data and materials}

All data generated or analyzed during this study are included in this published article.

\section{Authors' contributions}

FC, ZF and ZD initiated and designed the work, analyzed and interpreted the results, and wrote this manuscript. SY, JZ, PL 
and CY performed some experiments. All authors read and approved the final manuscript.

\section{Ethics approval and consent to participate}

For the use of human samples, the protocol for this study was approved by the Institutional Ethics Committee of Yunnan Tumor Hospital and all enrolled patients signed a written informed consent document.

\section{Patient consent for publication}

All patients within this study provide consent for the publication of their data.

\section{Competing interests}

The authors declare that they have no competing interests.

\section{References}

1. Chen AY, Jemal A and Ward EM: Increasing incidence of differentiated thyroid cancer in the United States, 1988-2005. Cancer 115: 3801-3807, 2009.

2. Liang L, Xu J, Wang M, Xu G, Zhang N, Wang G and Zhao Y: LncRNA HCP5 promotes follicular thyroid carcinoma progression via miRNAs sponge. Cell Death Dis 9: 372, 2018.

3. Gimm O: Thyroid cancer. Cancer Lett 163: 143-156, 2001.

4. Hundahl SA, Fleming ID, Fremgen AM and Menck HR: A National Cancer Data Base report on 53,856 cases of thyroid carcinoma treated in the U.S., 1985-1995 [see commetns]. Cancer 83: 2638-2648, 1998.

5. Liu B, Ye B, Yang L, Zhu X, Huang G, Zhu P, Du Y, Wu J, Qin X, Chen $\mathrm{R}$, et al: Long noncoding RNA $1 \mathrm{ncKdm} 2 \mathrm{~b}$ is required for ILC3 maintenance by initiation of Zfp292 expression. Nat Immunol 18: 499-508, 2017.

6. Zhu P, Wang Y, Wu J, Huang G, Liu B, Ye B, Du Y, Gao G, Tian Y, He L and Fan Z: LncBRM initiates YAP1 signalling activation to drive self-renewal of liver cancer stem cells. Nat Commun 7: 13608, 2016.

7. Wang Y, Gu J, Lin X, Yan W, Yang W and Wu G: lncRNA BANCR promotes EMT in PTC via the Raf/MEK/ERK signaling pathway. Oncol Lett 15: 5865-5870, 2018.

8. Yuan S, Liu Q, Hu Z, Zhou Z, Wang G, Li C, Xie W, Meng G, Xiang Y, Wu N, et al: Long non-coding RNA MUC5B-AS1 promotes metastasis through mutually regulating MUC5B expression in lung adenocarcinoma. Cell Death Dis 9: 450, 2018.

9. Zeng B, Lin Z, Ye H, Cheng D, Zhang G, Zhou J, Huang Z, Wang M, Cai C, Zeng J, et al: Upregulation of LncDQ is associated with poor prognosis and promotes tumor progression via epigenetic regulation of the EMT pathway in HCC. Cell Physiol Biochem 46: 1122-1133, 2018.

10. Chen X, Li D, Gao Y, Tang W, Iw L, Cao Y and Hao B: Long intergenic noncoding RNA 00152 promotes glioma cell proliferation and invasion by interacting with MiR-16. Cell Physiol Biochem 46: 1055-1064, 2018.

11. Wang R, Dong HX, Zeng J, Pan J and Jin XY: LncRNA DGCR5 contributes to CSC-like properties via modulating miR-330-5p/CD44 in NSCLC. J Cell Physiol 233: 7447-7456, 2018.

12. Yong S, Yabin Y, Bing Z, Chuanrong Z, Dianhua G, Jianhuai Z, Weidong Y, Shuming $W$ and Ling L: Reciprocal regulation of DGCR5 and miR-320a affects the cellular malignant phenotype and 5-FU response in pancreatic ductal adenocarcinoma. Oncotarget 8: 90868-90878, 2017.
13. Huang R, Wang X, Zhang W, Zhangyuan G, Jin K, Yu W, Xie Y, Xu X, Wang H and Sun B: Down-regulation of LncRNA DGCR5 correlates with poor prognosis in hepatocellular carcinoma. Cell Physiol Biochem 40: 707-715, 2016.

14. Livak KJ and Schmittgen TD: Analysis of relative gene expression data using real-time quantitative PCR and the 2(-Delta Delta C(T)) method. Methods 25: 402-408, 2001.

15. Ye B, Liu B, Yang L, Zhu X, Zhang D, Wu W, Zhu P, Wang Y, Wang S, Xia P, et al: LncKdm2b controls self-renewal of embryonic stem cells via activating expression of transcription factor Zbtb3. EMBO J 37: e97174, 2018.

16. Zhu P, Wang Y, Huang G, Ye B, Liu B, Wu J, Du Y, He L and Fan Z: lnc- $\beta$-Catm elicits EZH2-dependent $\beta$-catenin stabilization and sustains liver CSC self-renewal. Nat Struct Mol Biol 23: 631-639, 2016.

17. Esteller M: Non-coding RNAs in human disease. Nat Rev Genet 12: 861-874, 2011.

18. Lan X, Zhang H, Wang Z, Dong W, Sun W, Shao L, Zhang T and Zhang D: Genome-wide analysis of long noncoding RNA expression profile in papillary thyroid carcinoma. Gene 569: 109-117, 2015.

19. Wang C, Yan G, Zhang Y, Jia X and Bu P: Long non-coding RNA MEG3 suppresses migration and invasion of thyroid carcinoma by targeting of Rac1. Neoplasma 62: 541-549, 2015.

20. Gou Q, Gao L, Nie X, Pu W, Zhu J, Wang Y, Liu X, Tan S, Zhou JK, Gong Y, et al: Long noncoding RNA AB074169 inhibits cell proliferation via modulation of KHSRP-mediated p21 expression in papillary thyroid carcinoma. Cancer Res 78: 4163-4174, 2018.

21. Ding S, Qu W, Jiao Y, Zhang J, Zhang C and Dang S: LncRNA SNHG12 promotes the proliferation and metastasis of papillary thyroid carcinoma cells through regulating wnt $/ \beta$-catenin signaling pathway. Cancer Biomark 22: 217-226, 2018

22. Wang X, Lu X, Geng Z, Yang G and Shi Y: LncRNA PTCSC3/miR-574-5p governs cell proliferation and migration of papillary thyroid carcinoma via Wnt/ $\beta$-catenin signaling. J Cell Biochem 118: 4745-4752, 2017.

23. Li T, Yang XD, Ye CX, Shen ZL, Yang Y, Wang B, Guo P, Gao ZD, Ye YJ, Jiang KW and Wang S: Long noncoding RNA HIT000218960 promotes papillary thyroid cancer oncogenesis and tumor progression by upregulating the expression of high mobility group AT-hook 2 (HMGA2) gene. Cell Cycle 16: 224-231, 2017.

24. Sun W, Lan X, Wang Z, Dong W, He L, Zhang T and Zhang H: Overexpression of long non-coding RNA NR_036575.1 contributes to the proliferation and migration of papillary thyroid cancer. Med Oncol 33: 102, 2016.

25. Chen EG, Zhang JS, Xu S, Zhu XJ and Hu HH: Long non-coding RNA DGCR5 is involved in the regulation of proliferation, migration and invasion of lung cancer by targeting miR-1180. Am J Cancer Res 7: 1463-1475, 2017.

26. Wang Y, Zeng X, Wang N, Zhao W, Zhang X, Teng S, Zhang Y and $\mathrm{Lu} \mathrm{Z}$ : Long noncoding RNA DANCR, working as a competitive endogenous RNA, promotes ROCK1-mediated proliferation and metastasis via decoying of miR-335-5p and miR-1972 in osteosarcoma. Mol Cancer 17: 89, 2018.

27. Wang Z, Zhang H, Zhang P, Li J, Shan Z and Teng W: Upregulation of miR-2861 and miR-451 expression in papillary thyroid carcinoma with lymph node metastasis. Med Oncol 30: 577,2013

28. Zhao M, Li L, Zhou J, Cui X, Tian Q, Jin Y and Zhu Y: MiR-2861 behaves as a biomarker of lung cancer stem cells and regulates the HDAC5-ERK system genes. Cell Reprogram 20: 99-106, 2018.

29. Xu J, Wan X, Chen X, Fang Y, Cheng X, Xie X and Lu W: miR-2861 acts as a tumor suppressor via targeting EGFR/AKT2/CCND1 pathway in cervical cancer induced by human papillomavirus virus 16 E6. Sci Rep 6: 28968, 2016. 